\title{
Global crises challenge environmental science and biotechnology
}

\author{
P. A. Wilderer
}

Published online: 16 October 2009

(C) Springer Science+Business Media B.V. 2009

In 1960, about 3 billion people lived on Earth. Forty years later, we were 6 billion, and by 2040 we might be as many as 9 billion. We are many, indeed; some assume that we are already too many with respect to the Earth system's capacity to supply humanity with indispensable resources, and to regenerate and absorb the wastes we are generating. By the way: What is the bearing capacity of our planet? Is it 200 million people as Lovelock (2009) suggests, or 650 million proposed by and Gorshkov et al. (2000)? According to Wackernagel (WWF 2008) humanity used in 2008 the equivalent of 1.3 planets considering the resources we need or believe to need, and considering the waste we emit. However, as everybody knows, we have only one planet at our disposal.

More people require additional energy, food and water. More land is also required for people to settle, to produce agricultural products including energy crops, to operate industrial installations and airports, and to permit road traffic. Turning to the back side of the coin, more people generate more solid, liquid and gaseous wastes, which are typically sent away and out of sight causing agricultural land, water bodies and the air to deteriorate in quality. This, in turn, aggravates supply of people with the required base of life, qualitatively and quantitatively alike.

P. A. Wilderer $(\square)$

Schliersee, Germany

e-mail: peter.wilderer@mytum.de
There is one additional factor to be considered. Increase of population density beyond a certain threshold bears the risk of societies to destabilize because individuals lose the territorial freedom they naturally claim. Like most other species on Earth, people get aggressive when a minimum of space around them is trespassed by strangers. Maternal protectiveness, territorial defense, egoism, greed and herd instinct are genetically encoded properties of human beings-transcribed to family networks, tribes, economic institutions and political systems.

In conclusion, the primary problem we are facing is the rapid increase of the population density, globally as well as locally. We have to deal with a population density crisis, and we ought to find measures to handle this crisis. Derived from the population density crisis, we face a complex of secondary, widely interwoven crises such as the climate, water, food, poverty, social inequality and economy. Because all these sub-crises are interrelated, any attempt to solve a single sub-crisis cannot lead to a sustainable solution. It only might provide us with some time to relax. What we ought to do is to take a multi-dimensional inter- and trans-disciplinary approach taking into account local and regional peculiarities with respect to cultural heritage, religious concerns, and tribal traditions. Starting from top to bottom, we are supposed to make any attempt to minimize the impacts originating from densely populated areas, and their effects on the life enabling function of the ecosystems. The term Earth System 
Engineering (ESE) describes measures to be taken. ESE is defined as the deliberate, planned and well controlled intervention into the Earth system with the intention to preserve the life supporting function of the Earth system including all its components such as the lithosphere, the hydrosphere, the atmosphere, the biosphere and the anthroposphere.

Centuries ago, an early example of population density crisis threatened the ancient city cultures, and lead to the development and implementation of mitigation and adaptation measures. For instance, long distance aqueducts were built to transport water into, and sewers were laid out to transport stormwater runoff and wastewater away from the city centres. In this context, the term "mitigation" stands for policies to reduce through technical means water shortages, flooding and accumulation of mud in cities, and the term "adaption" stands for education of the population to deal consciously with a limited resource: water.

In the nineteenth century population density increased significantly within the rapidly growing industrial regions in England and central Europe. Concomitantly, the local demand of water, food, housing and health care grew as well as frictions among the workers and labor unrests caused by unfair working hours and wages. Additional mitigation and adaption measures had to be introduced. It was the advent of labor unions, a classical mitigation instrument on the societal level. Hygiene science developed and, as a result, people were trained to wash hands, a metaphor of adaption to a hygienically safe lifestyle. Waterworks were developed and installed to guarantee supply of hygienically safe water. Simultaneously, flushing toilets, flushing sewers and wastewater treatment plants were introduced as a contribution to hygienic safety, and later on to maintain a good ecological status of aquifers, rivers, lakes and coastal zones. All those measures might be rated as a contribution to mitigate impacts of population density increases.

Today, it is not only the global population but, more dramatically, the aggregation of people in urban areas which is increasing. More than half of the world's population lives in cities, and almost $80 \%$ of people settle in a $50 \mathrm{~km}$ wider stripe along the sea coast. People migrate to cities in the expectation of finding jobs and a better life-whatever that means. Problems resulting from the increase of population density in cities are sky rocketing. Which measures do we have to develop and implement to manage the contemporary and the future population density crisis? Which role shall environmental bio-technology play?

In future, migration and aggregation of people is likely to reach an unprecedented dimension caused by rising sea level and loss of habitable land along the coast lines-where already most of the world's population live (see above). Here, the climate change crisis comes into play, and the reasons which lead to global warming.

We have learned that extensive emission of carbon dioxide over the past 100 years has caused the surface temperature of Earth to rise. Most probably, it is not only the increase of the amount of $\mathrm{CO}_{2}$ in the atmosphere which triggers global warming, but also the increase of several other so called greenhouse gases including methane and water vapor. The latter has not received much attention in the public debate although water vapor is known to be the strongest greenhouse gas of all. The water vapor content has increased because of the warming of the oceans. Under conditions undisturbed by mankind, the water vapor as well as the $\mathrm{CO}_{2}$ partial pressure would likely be controlled by processes inherent in large ecosystems such as tropical and boreal rain forests (Gorshkov et al. 2000). According to the Gaia theory proposed by Lovelock (1979), the controlling function of highly diversified ecosystems has been responsible for the preservation of liquid water on Earth over more than 3 billion years, and for a temperature level which permits persistence of psychrophilic and mesophilic life forms on Earth. Nevertheless, with reallocation of land formerly occupied by forests and wetlands, the controlling function of ecosystems gets more and more lost. Global warming, spread of drought situations, desertification, melting of glaciers, melting of the ice cap of Antarctica and Greenland, and the subsequent rise of the sea level are among the consequences (Lovelock 2006). Recent observations suggest that loss of polar ice and rise of the sea level proceeds much faster than predicted by the IPCC models. It appears that we will lose within the twenty-first century already major fractions of habitable land forcing billions of people to migrate to areas which remain habitable- "lifeboats" as Lovelock (2009) calls them. These areas will get tremendously overcrowded and here we are back to what we have discussed above: population density at an unprecedented level, and the resulting population density crisis. 
In the current public debate, the consequences of overpopulation of our future "lifeboats" are rarely discussed. It appears that the world is paralyzed by the $\mathrm{CO}_{2}$ issue. Almost all efforts are directed to the diminishing of $\mathrm{CO}_{2}$ emissions. This is certainly worth an effort, but will it solve the problems which we will face in the very near future? Will geo-engineeringthe deliberate large-scale interventions into the Earth-Sun system with the aim to control the balance between incoming solar radiation and outgoing infrared radiation-save us from the societal collapse? Geo-engineering might be considered an inevitable temporary rescue measure in a world of conflicting interests. We should not concentrate on curing symptoms, however. The real question to be addressed in science and technology is: How can we prepare mankind to arrange itself in overcrowded "lifeboat" situations? In an editorial of the journal Science, Reid et al. (2009) brought it to the point: "The major challenge today includes the need to understand complex interrelationships among biological, geochemical, climate and social systems; the consequences of global change for the Earth system and society and the feedback loops involved; and the science of mitigation, adaption and sustainability. Natural science should no longer dictate the Earth system research agenda; social sciences will be at least as important in its next phase". It appears that we ought to put human obligations before human rights (Lovelock 2009).

What does all this mean for environmental science and biotechnology? It is our duty as scientists and engineers to act as governors of public health and well-being. Based on the inherited knowledge and the current state of technology, we are supposed to develop methods which will fit in the "lifeboat" scenario of the future. We have to make sure that water in sufficient quantity and quality is available for people, industry and agriculture in overcrowded settings. Most likely, this task can only be met by making use of alternative sources of water including wastewater and atmospheric humidity (Wilderer 2010). In some parts of the world, wastewater is already accepted as a potential source of service water, even as drinking water (Tortajada 2006). Various options are available to access atmospheric humidity in the form of vapor, fog or clouds. Water vapor can be abstracted from the atmosphere by means of condensation processes. Fog can be captured by means of net-like structures. More complicated is triggering artificial rainfall, but it is fair to assume that advances in atmospheric physics and meteorology will soon provide the basic knowledge required to develop technologies which are more reliable and more efficient than methods such as cloud seeding. Controlled electric charging of water molecules and droplets in clouds appears to be a promising future method of rainfall generation. Capturing and safe storage after effective storage of precipitation are methods to be further developed.

Water reuse and abstraction of atmospheric humidity are just two examples of technologies to be put in focus, but not by natural scientists and process engineers alone. Risk analysis and risk management as well as participative technology assessment-assessment in close cooperation with the end-users including the civil society in "lifeboat" environments - are areas of research and development which have to be treated as integral parts of the whole business. Above all, we are committed to find innovative approaches of coexistence of humanity and large-scale ecosystems. It is unrealistic to assume that technology might ever be capable of replacing ecosystem function. In many respects, humans are certainly superior over animals. However, to be able to master the upcoming complex of crises we ought to aim our intelligence, our ability to accumulate and transfer from generation to generation knowledge and experience at sustainable survival not of the individual but of the whole, and evolve to a state of harmony with the Earth as a living planet.

\section{References}

Gorshkov VG, Gorshkov VV, Makarieva AM (2000) Biotic regulation of the environment. Springer Praxis Publishing, Chichester

Lovelock J (1979) A new look of life on Earth. Oxford University Press, Oxford

Lovelock J (2006) The revenge of Gaia. Penguin Books, Baltimore

Lovelock J (2009) The vanishing face of Gaia. Basic Books, New York, USA

Reid WV, Bréchignac C, Lee YT (2009) Earth system research priorities. Science 325:245-246 
Tortajada C (2006) Water management in Singapore. Water Resour Dev 22:227-240

Wilderer PA (2010) Abstraction of atmospheric humidity. In: Wilderer PA (ed) Treatise of water science. Elsevier, Amsterdam (In press)
WWF (2008) Living planet report 2008. World Wide Fund for Nature, Gland, Switzerland 